\section{EMERGENCY PREPAREDNESS}

\section{Friday 29 August-Concurrent Session 6}

Secrets of the Bali Fellowship: Disaster proofing your Emergency Department during a disaster

\section{Deidre Tame}

In the last 10 years there has been a significant increase in disasters, both natural and manmade. Recent terrorist and epidemic events have underscored the potential for large numbers of casualties flooding the health system. The stark reality that a disaster is "locally owned" for the immediate duration has been affirmed and emphasized. Few surplus resources exist in our current health care system to accommodate casualties of mass disaster. Therefore, the impact of surge, during an incident, has the potential to be devastating. Due to Western Australia's small number of health provider's and their geographical isolation, recognition of this problem and planning for it, is crucial.

On a day-to-day basis Australian Hospitals operate at $100 \%$ capacity with overcrowded emergency departments, complicated by problems of bed block. In event of a major incident the ability to absorb surges of casualties into the health system, would be significantly limited by these factors. Further more, controlling the flow of these patients in areas of high demand, requires planning and flexible guidelines. Currently plans for managing bottlenecking in patient flow when dealing with casualty surge are limited and not evidenced-based.

This presentation explores the secrets of surge capacity and decreasing the problems of bottlenecking by improving patient flow in an Emergency department during a disaster. The presentation draws on the experience of nine different hospitals in five different countries who have been at the fore front of disaster management and have experienced and addressed problems with surge capacity, streaming and prioritisation of patient flow.

doi:10.1016/j.aenj.2008.09.047

Reflections on emergency preparedness level of selected Asian hospitals visited during Bali Fellowship study tour

Ian Williams

Emergency Preparedness Unit, Fremantle Hospital, Fremantle, WA, Australia

My presentation is based on the report of a study tour undertaken in 2005-2006 to review disaster preparedness of hospital systems in the major Asian cities of Hong Kong, Singapore, Taipei, Tokyo and Kobe.

My proposal was to visit some major Asian cities that had been affected by a significant major incident in the last 10 years to see how well prepared their health systems were. What were the lessons learned? What changes have been made? Are they better prepared as a result of their experiences?

The focus of my visit was on the key areas of Chemical, Biological, Radiological Nuclear (CBRN) response, Hospital
Emergency Incident Command System (HEICS) and Severe Acute Respiratory Syndrome (SARS).

It has often cynically been said that the level of interest in disaster management issues is inversely proportional to the length of time since the last major event. I had wanted to investigate how these events had affected and shaped the preparedness in some of these countries. In Australia geography and good fortune have by and large spared us events on the scale that many of our Asian neighbours have endured, however we cannot afford to become complacent.

My experiences were both strategic and operational view points of practitioners in the countries that I visited.

doi:10.1016/j.aenj.2008.09.048

The threat to Emergency Departments from bioterrorism in Australia

John Bates

\section{Public Health Microbiology Laboratory, QHFSS, Australia}

The Commonwealth Department of Health (DoHA) has recently developed two lists of threat agents in consultation with relevant stakeholders around Australia. These have been labeled Tier 1 and Tier 2 agents. This list includes bacteria, viruses, and toxins derived from bacteria and plants. Legislation will soon be introduced to introduce controls on laboratories who store these agents. This presentation will look at the agents on these two lists and examine those that represent the biggest threat in the Australian context, detailing their clinical presentation, their mode of spread and how they might be used as bioterrorist agents. A number of these agents produce flu-like symptoms initially, making diagnosis difficult. This has important ramifications for patients, who will die unless antibiotic therapy is initiated rapidly. The discussion will concentrate on the threat theses agents pose to emergency staff when receipting patients exposed to a deliberate release of agent. An attack of this nature would quickly overwhelm even large Emergency Departments, not only from presentations of seriously ill patients but also from the large numbers of worried well who will present. Whether the agent is infectious or not will have serious ramifications for ongoing spread of the agent throughout the community.

doi:10.1016/j.aenj.2008.09.049

Major incident preparedness: A hospital review of a CBR exercise-lessons learnt!

\section{Diana Patton}

Emergency Department, Monash Medical Centre, Clayton, Melbourne, VIC, Australia

Monash Medical Centre is situated on the outskirts of Melbourne, is a tertiary referral hospital and boasts a new purpose built Emergency Department.

The opportunity existed for the emergency department to test its new decontamination facilities and the hospital to test its ability to respond to a Major incident. Exercise November involved a collaborative and multidisciplinary 